Alana Patrício Stols Cruzeta ${ }^{1}$

Ana Claudia Lawless Dourado ${ }^{1}$

Maria Tereza Mattos Monteiro ${ }^{1}$

Rafael Olívio Martins ${ }^{1}$

Talita Aparecida Calegario ${ }^{1}$

Dayani Galato ${ }^{1}$

\title{
Fatores associados à compreensão da prescrição médica no Sistema Único de Saúde de um município do Sul do Brasil
}

\author{
Factors associated with the understanding of medical prescriptions \\ in the Unified Health System in a city in southern Brazil
}

${ }^{1}$ Programa de PósGraduação em Ciências da Saúde, Universidade do Sul de Santa Catarina. Av. José Acácio Moreira 787, Dehon. 88.704-900 Tubarão SC Brasil.

dayani.galato@gmail.com

\begin{abstract}
A cross-sectional study via interviews was conducted in the city of Tubarão in the state of Santa Catarina in order to identify the association between prescription indicators and the profile of the healthcare users and their understanding of medical prescriptions among patients attended by the Unified Health System. Information was collected on the profile of the healthcare users, the prescription indicators recommended by the World Health Organization and the understanding of drug prescriptions. Three hundred subjects were interviewed and the associated factors were identified using the Chi-square test. Of the respondents, $59.3 \%$ were able to read the prescription or knew the drug names, $80.7 \%$ knew the drug indication, $73.7 \%$ knew the dosage, $72.3 \%$ knew the dosage intervals and $72.7 \%$ knew the duration of treatment. However, only $46.3 \%$ of the respondents fully understood the prescription. Between the prescriptions indicators only the number of drugs in prescriptions was significantly associated with understanding. On the other hand, having up to eight years of schooling, belonging to socioeconomic class C1, being under 49 years of age and being female were significantly associated with the understanding of the drug prescription.
\end{abstract}

Key words Understanding, Drug prescription, Unified Health System, Prescription indicators, Drugs, Drug use
Resumo Com o objetivo de identificar a associação entre os indicadores de prescrição e o perfil do paciente à compreensão de prescrições de medicamentos de pacientes atendidos no Sistema Único de Saúde do município de Tubarão (Santa Catarina), foi realizado um estudo transversal por meio de entrevista. Coletou-se informações sobre o perfil dos usuários do Serviço, indicadores da prescrição médica da Organização Mundial da Saúde e sobre a compreensão da prescrição. Os fatores associados foram identificados através do teste quiquadrado. Foram entrevistados 300 sujeitos. Entre estes, 59,3\% conseguiam ler a prescrição ou sabiam o nome dos medicamentos, 80,7\% conheciam a indicação, 73,7\% sabiam a dose, 72,3\% sabiam a frequência entre as doses e 72,7\% conheciam a duração do tratamento. No entanto, compreenderam totalmente a prescrição $46,3 \%$ dos entrevistados. Verificou-se que dos indicadores de prescrição apenas o número de medicamentos está associado à compreensão, por outro lado, a idade do paciente menor que 49 anos, ter estudado mais que oito anos, pertencer a classe econômica C1 ou superior e ser mulher associam-se de forma significativa com a compreensão da prescrição.

Palavras-chave Compreensão, Prescrição de medicamentos, Sistema Único de Saúde, Indicadores de prescrição, Medicamentos, Uso de medicamentos 


\section{Introdução}

A prescrição médica é um instrumento essencial para a indicação da terapêutica, devendo conter informações de identificação do paciente e do tratamento a ser utilizado, informando a dose, a frequência e a duração do tratamento adequado ao perfil e a condição de saúde do paciente ${ }^{1-3}$. A falta de compreensão da prescrição é considerada uma importante razão para a não adesão ao tratamento medicamentoso ${ }^{4,5}$.

Problemas na compreensão das informações da prescrição são comuns nos serviços de saúde e suas causas podem ser atribuídas a uma linguagem complexa, informações desorganizadas ou implícitas e uma caligrafia inadequada, as quais tornam ilegível a informação escrita ${ }^{6}$. Contudo, supõe-se que outros fatores como a quantidade de medicamentos, as classes farmacológicas, as forma farmacêutica e os nomes dos medicamentos (se genérico ou não) presentes na prescrição poderiam influenciar na sua compreensão.

Muitos destes fatores são medidos através dos indicadores do uso de medicamentos desenvolvidos pela Organização Mundial da Saúde $(\mathrm{OMS})^{5}$. Estes indicadores de prescrição permitem conhecer as práticas terapêuticas correntes, comparar parâmetros entre instituições similares e descrever as necessidades de medicamentos da população atendida, assim como identificar o uso abusivo de antibióticos, quantificar prescrições contendo medicamentos genéricos, através da análise da prescrição médica ${ }^{1,7-10}$.

Estudos como o de Fröhlich e Mengue ${ }^{10}$ tem se centrado em avaliar os indicadores de qualidade das prescrições e a propor novos indicadores. Neste trabalho ${ }^{10}$, mesmo que os autores tenham analisado a opinião dos pacientes sobre alguns aspectos da prescrição, não foi avaliada a compreensão da prescrição.

Contudo, além da própria prescrição fatores relacionados com o paciente também podem influenciar no conhecimento sobre os medicamentos prescritos. Desta forma, levantando-se a hipótese de que os indicadores de prescrição propostos pela OMS ${ }^{5}$ poderiam interferir juntamente com características dos pacientes na compreensão da prescrição, foi realizado este trabalho com o objetivo de identificar a associação entre estes fatores à compreensão de prescrições de medicamentos de usuários do Sistema Único de Saúde do município de Tubarão, Santa Catarina.

\section{Métodos}

Estudo epidemiológico de delineamento transversal, mediante a aplicação da técnica da entrevista. O município estudado (Tubarão) está situado a 150 quilômetros ao Sul da capital do Estado de Santa Catarina (Florianópolis). Esta cidade possuía 96.529 habitantes segundo dados da estimativa populacional para o ano de $2009^{11}$. A população do estudo foi representada por usuários do Sistema Único de Saúde do município em estudo ou por seus cuidadores que aceitaram participar da pesquisa.

Para o cálculo da amostra foi adotado um erro de 5\%, um nível de $95 \%$ de confiança e uma prevalência esperada de $25 \%$, conforme dados de Silva et al. ${ }^{2}$ que apresentaram resultados de compreensão entre 20 e $30 \%$. A amostra foi calculada para 288 sujeitos, no entanto, a mesma foi aumentada para 300, em função de uma orientação da Organização Pan-Americana de Saú$\mathrm{de}^{12}$ que recomenda avaliar 30 prescrições por Unidade de Saúde para determinar os indicadores de prescrição.

Para composição da amostra foram selecionadas 10 (dez) Unidades Básicas de Saúde (UBS) distribuídas geograficamente adotando-se os pontos cardeais, sendo que o município possuía no período da pesquisa 21 UBS. As UBS foram visitadas em dias alternados, sendo entrevistados pacientes ou cuidadores até completar a amostra de 300 entrevistas (30 por Unidade).

A coleta foi realizada entre os meses de fevereiro e maio do ano de 2009 por dois entrevistadores previamente treinados. O usuário do Serviço ou em caso de menores, seu cuidador, foi abordado na saída do consultório médico para a apresentação da pesquisa. Aceitando participar o mesmo foi convidado a assinar o termo de consentimento livre e esclarecido e a responder questões a respeito de sua prescrição.

$\mathrm{O}$ instrumento adotado possibilitou a coleta de dados relacionados ao perfil dos entrevistados (idade, sexo, escolaridade, classificação econômica), à compreensão da prescrição, aos medicamentos prescritos e aos indicadores de prescrição OMS .

A Classificação econômica adotada foi aquela sugerida pela Associação Brasileira das Empresas de Pesquisa (Critério Brasil) ${ }^{13}$, que classifica os entrevistados em extratos de A (uma renda familiar estimada acima de 20 salários míni- 
mos) ao E (menor que um salário mínimo). Esta classificação enfatiza a estimativa do poder de compra das pessoas através da análise de posse de itens.

A compreensão da prescrição (variável de desfecho) foi determinada através do conhecimento dos pacientes a respeito dos medicamentos que os mesmos deveriam utilizar presentes na prescrição, considerando-se que o entrevistado compreende a prescrição quando o mesmo sabe o nome do medicamento (ou o lê), conhece sua indicação, dose, intervalo entre doses e duração do tratamento ${ }^{2,14}$.

Os indicadores de prescrição da OMS selecionados foram: o número de medicamentos por prescrição; percentual de medicamentos por nome genérico; percentual pertencente às listas padronizadas, neste caso, Relação Nacional de Medicamentos Essenciais (RENAME) e Relação Municipal de Medicamentos Essenciais (REMU$\mathrm{ME})$ e; percentual de prescrições contendo medicamentos antimicrobianos ${ }^{5}$.

Os medicamentos prescritos foram classificados de acordo com a Anatomical Therapeutic Chemical (ATC).

Destaca-se que foi realizado um pré-teste ( $n$ =10) para avaliação da aplicabilidade do instrumento de coleta de dados e da técnica de abordagem dos usuários do serviço. Processo realizado em uma Unidade de Saúde de outro Município, cujos dados não foram incluídos nesta pesquisa.

As informações coletadas foram inseridas em um banco de dados no programa EpiData $3.0 \mathrm{e}$ analisados com o auxílio do programa do SPSS 19.0. Foram realizadas análises descritivas adotando-se as frequências e as proporções para as variáveis nominais e as medidas de tendência central e dispersão para as variáveis numéricas. A prevalência de compreensão foi estimada com um Intervalo de Confiança de 95\% (IC 95\%).

Realizou-se o teste de qui-quadrado e estimou-se a razão de prevalência (RP) com intervalo de confiança de $95 \%$ para avaliar a associação entre variável de desfecho (compreensão) e as de perfil dos entrevistados e de indicadores de prescrição. Quando necessário para a análise, as variáveis numéricas foram reagrupadas tendo como base a mediana.

Este projeto foi aprovado pelo Comitê de Ética em pesquisa da Universidade do Sul de Santa Catarina.

\section{Resultados}

Os resultados mostram que das 300 entrevistas realizadas, $85,0 \%$ foram realizadas com os pacientes. Dos entrevistados 66,3\% foram mulheres e a média de idade dos sujeitos foi de 48,0 ( \pm 17,3) anos. A renda obtida variou de A2 a E, sendo as mais prevalentes a C1 $(28,7 \%)$, seguido de D $(23,3 \%)$, B2 $(23,0 \%)$ e C2 $(21,3 \%)$.

Quando investigado a distribuição da escolaridade por sexo, observa-se que $66,8 \%$ das mulheres e $84,2 \%$ dos homens estão classificados no grupo com menor escolaridade (até oito anos de estudo).

Foram prescritos ao todo 561 medicamentos, sendo que o número por prescrição variou de um a 11 , com média de $1,87( \pm 1,31)$ e mediana de um.

Destes medicamentos, observou-se que as classes farmacológicas mais prevalentes, segundo a ATC, foram: Sistema cardiovascular $(33,7 \%)$ destacando-se o captopril $(8,4 \%)$ e enalapril $(2,7 \%)$; Trato alimentar e metabolismo (19,5\%) sendo a glibenclamida $(5,1 \%)$ e metformina $(4,7 \%)$ as mais descritas; Sistema nervoso $(17,2 \%)$ tendo o paracetamol $(7,8 \%)$ como principal representante e; Sangue e órgãos hematopoiéticos $(10,7 \%)$ destacando-se o ácido acetilsalicílico (10,0\%).

A Tabela 1 apresenta os critérios utilizados para medir a compreensão da prescrição com seus respectivos resultados, sendo observada uma prevalência de compreensão de 46,3\% (IC 95\%: 40,6-52,2).

A Tabela 2 apresenta a análise entre a variável de desfecho, compreensão, e as demais variáveis do estudo.

Tabela 1. Distribuição dos critérios utilizados para a avaliação da compreensão das prescrições por parte dos entrevistados (Tubarão/SC - 2009).

\begin{tabular}{lc}
\hline \multicolumn{1}{c}{ Critérios } & $\begin{array}{c}\text { Resultados } \\
\mathbf{n}(\%)\end{array}$ \\
\hline $\begin{array}{l}\text { Consegue ler ou sabe o nome do } \\
\text { medicamento }\end{array}$ & $178(59,3)$ \\
Conhece indicação & $242(80,7)$ \\
Sabe a dose & $221(73,7)$ \\
Sabe a frequência entre as doses & $217(72,3)$ \\
Conhece a duração do tratamento & $218(72,7)$ \\
Compreende a prescrição & $\mathbf{1 3 9}(\mathbf{4 6 , 3 )}$
\end{tabular}


Tabela 2. Associação das variáveis de caracterização dos entrevistados e dos indicadores da OMS com a compreensão da prescrição.

\begin{tabular}{|c|c|c|c|c|}
\hline Variável & $\begin{array}{l}\text { Total } \\
\mathbf{n}(\%)\end{array}$ & $\begin{array}{c}\text { Compreensão } \\
\mathbf{n}(\%)\end{array}$ & $\begin{array}{c}\text { RP } \\
(\text { IC 95\%) bruta }\end{array}$ & $p$ \\
\hline Sexo & & & & 0,008 \\
\hline Feminino & $199(66,3)$ & $103(51,8)$ & $1,45(1,08-1,95)$ & \\
\hline Masculino & $101(33,7)$ & $36(35,6)$ & 1,00 & \\
\hline Escolaridade & & & & $<0,001$ \\
\hline$\leq$ oito anos & $218(72,7)$ & $78(35,8)$ & $0,48(0,39-0,60)$ & \\
\hline$>$ oito anos & $82(27,3)$ & $61(74,4)$ & 1,00 & \\
\hline Idade & & & & $<0,001$ \\
\hline Até 49 anos & $155(51,7)$ & $93(60,0)$ & $1,89(1,44-2,48)$ & \\
\hline 50 anos ou mais & $145(48,3)$ & $46(31,7)$ & 1,00 & \\
\hline Renda & & & & $<0,001$ \\
\hline Até C1 & $165(55,0)$ & $103(62,4)$ & $2,34(1,73-3,17)$ & \\
\hline C2 ou menor & $135(45,0)$ & $36(26,7)$ & 1,00 & \\
\hline Número de medicamentos por prescrição & & & & 0,044 \\
\hline Até um & $167(55,7)$ & $86(51,5)$ & $1,29(1,01-1,67)$ & \\
\hline Dois ou mais & $133(44,3)$ & $53(39,8)$ & 1,00 & \\
\hline Medicamentos prescritos por genérico & & & & 0,274 \\
\hline Sim & $443(91,7)$ & $182(41,1)$ & 1,00 & \\
\hline Não & $40(8,3)$ & $20(50,0)$ & $1,22(0,88-1,69)$ & \\
\hline Prescrição pela REMUME & & & & 0,364 \\
\hline Sim & $443(81,0)$ & $187(42,2)$ & 1,00 & \\
\hline Não & $104(19,0)$ & $49(47,1)$ & $1,12(0,89-1,41)$ & \\
\hline Prescrição pela RENAME & & & & 0,426 \\
\hline Sim & $461(84,1)$ & $196(42,5)$ & 1,00 & \\
\hline Não & $87(15,9)$ & $41(47,1)$ & $1,11(0,87-1,42)$ & \\
\hline Prescrição contendo antibiótico & & & & 0,311 \\
\hline Sim & $41(3,7)$ & $22(53,7)$ & $1,19(0,87-1,63)$ & \\
\hline Não & $259(86,3)$ & $117(45,2)$ & 1,00 & \\
\hline
\end{tabular}

\section{Discussão}

O uso dos medicamentos deve seguir o princípio do uso racional proposto pela $\mathrm{OMS}^{15}$, onde se pressupõem que além da seleção adequada, o medicamento deverá ser usado na posologia e no período de tempo adequados. Neste contexto, a compreensão da prescrição torna-se uma condição fundamental para o uso racional dos medicamentos.

No entanto, os resultados desta pesquisa apontam que menos da metade dos entrevistados compreendiam as prescrições, levando em consideração todos os critérios investigados. Entre estes, observa-se que o pior avaliado foi o consegue ler ou sabe o nome do medicamento, dado bastante crítico, uma vez que os pacientes poderiam consultar a prescrição. Um dos empecilhos para este critério pode ser a letra do médico, já que no município em estudo não está implantado no Serviço
Público de Saúde a prescrição eletrônica, implantação esta que poderia minimizar este problema ${ }^{16}$. Outro fator que pode ter influenciado este fato é a baixa escolaridade dos entrevistados.

Os dados relacionados à compreensão da dose e do intervalo são muito semelhantes aos de outro estudo ${ }^{2}$, no qual cerca de $20 \%$ dos pacientes entrevistados demonstraram não saber a dose e $30 \%$ o intervalo de administração dos medicamentos contidos na prescrição. Isto ainda é mais relevante quando se identificou naquela pesquisa ${ }^{2}$ que a maioria dos pacientes já estava utilizando os medicamentos prescritos, em grande parte de uso contínuo, como também é observado no presente trabalho.

Confirmando a informação da maior prevalência de medicamentos de uso continuo, observa-se entre as classes farmacológicas, identificadas de acordo com a ATC, principalmente medicamentos que atuam no Sistema Cardiovascular e no Trato Alimentar e Metabolismo, o que tam- 
bém tem sido observado em outros estudos ${ }^{2,17,18}$.

A falta de informação sobre a duração do tratamento, observada em quase 30\% dos entrevistados também merece discussão, pois pode levar os pacientes à interrupção precoce ou à prorrogação do tratamento, com a possibilidade de causar problemas relacionados à efetividade ou à segurança dos medicamentos, respectivamente ${ }^{16}$.

Quando analisada a compreensão da prescrição com o perfil da mesma, através dos indicadores da OMS (Tabela 2), observou-se que o número de medicamentos esteve associado à compreensão. Segundo Acúrcio et al. ${ }^{18}$, a alta complexidade do tratamento dificulta a sua compreensão, pois em prescrições com menor número de medicamentos há menos informações para ser assimilada e, consequentemente, a sua compreensão é favorecida ${ }^{10}$.

Outros indicadores de prescrição não se associaram à compreensão da prescrição. Entre estes, destaca-se a importância e a obrigatoriedade da prescrição por nome genérico no Sistema Único de Saúde ${ }^{19}$. A adoção da nomenclatura genérica traz vantagens por diminuir a possibilidade de erros de medicação na dispensação e por reduzir o custo dos medicamentos quanto da necessidade de aquisição por parte do paciente ${ }^{18}$. No entanto, a prescrição por esta nomenclatura pode interferir na compreensão da mesma, pelo fato de geralmente os nomes genéricos dos medicamentos serem mais complicados que os comerciais, em especial, para aqueles pacientes que usam medicamentos contínuos já prescritos em algum momento pelo nome comercial. Contudo, esta dificuldade não foi observada.

Também se destaca que a presença de antibióticos na prescrição não altera a compreensão da mesma. Este é um fato preocupante, pois esta classe de medicamentos além de estar relacionado à recuperação da saúde do próprio paciente, seu uso inadequado pode ocasionar a seleção de cepas resistentes o que pode tornar-se um problema de saúde pública ${ }^{20,21}$.

Quanto ao perfil dos entrevistados, observou-se que o sexo feminino está associado á compreensão. Esta maior compreensão da prescrição pode ser explicada por diversos fatores, entre eles, a maior escolaridade observada entre as mulheres, o fato de utilizarem mais os serviços de saúde ${ }^{19}$, além de usarem mais medicamentos e trazerem para si a responsabilidade dos mesmos perante a família.

Outro dado relacionado ao perfil dos entrevistados que contribui para a compreensão da prescrição foi à idade, com as pessoas mais jo- vens compreendendo melhor do que as mais velhas. Este fato pode estar relacionado à maior escolaridade dos mais jovens. No entanto, gera um paradoxo, pois, se por um lado os adultos mais jovens tem maior grau de instrução o que representa maior possibilidade de compreensão da prescrição, por outro lado, os mais velhos geralmente utilizam medicamentos de uso contínuo, o que também deveria estar associada à esta questão.

Neste sentido, a maior escolaridade associouse de forma significante. Ou seja, pessoas com menos de oito anos de escolaridade compreendem menos a prescrição do que pessoas com maior escolaridade, isto pode ser explicado pela dificuldade na leitura (parâmetro ligado diretamente ao grau de escolaridade). Outro fator que deve estar relacionado à maior compreensão é a maior aproximação dos mais letrados com a linguagem utilizada pelos prescritores. Fröhlich et al. ${ }^{22}$ também identificaram em sua pesquisa que a escolaridade esteve associada com o conhecimento da prescrição.

Já a menor classificação socioeconômica além de estar geralmente relacionada à menor escolaridade, geralmente promove o adoecimento mais precoce fazendo com que estas pessoas estejam mais propensas ao maior número de problemas de saúde, consequentemente a uma maior utilização de medicamentos ${ }^{23}$, o que também pode estar associado a uma menor compreensão da prescrição ${ }^{9,18}$.

Esta falta de compreensão a respeito da terapêutica prescrita pode ter diversas consequências, entre elas destacam-se os retornos precoces aos consultórios, a realização de novos exames, o agravamento de quadros e o surgimento de possíveis sequelas 9 . Isso não traz prejuízos apenas ao paciente, mas também à comunidade ${ }^{9,24,25}$.

Cita-se como limitações deste estudo o fato de não ter sido investigado o recebimento por parte dos entrevistados de orientações do prescritor sobre os medicamentos prescritos, bem como a avaliação da legibilidade das prescrições.

\section{Conclusão}

A associação da compreensão da prescrição com indicadores da OMS só pôde ser observada com o número de medicamentos constantes na mesma. No entanto, observou-se estar fortemente associado a diversas variáveis de perfil dos pacientes, como sexo feminino, possuir mais de oito anos de estudo, ter idade até 49 anos e ser de 
classificação socioeconômico $\mathrm{C} 1$ ou superior.

Nesse sentido, este estudo contribui para demonstrar a necessidade de ampliar medidas estruturais que minimizem dificuldades de compreensão da prescrição. Entre estas medidas, destaca-se a implantação de prescrição eletrônica, a redução da complexidade dos tratamentos quando possível e maior atenção por parte dos profissionais da saúde (prescritores e dispensadores de medicamentos) no repasse de informações que garantem o uso racional de medicamentos, em especial, aos pacientes mais carentes e com menor grau de instrução.

\section{Colaboradores}

APS Cruzeta participou da análise dos dados e da redação final do trabalho e análise critica da versão final; TA Calegario participou da concepção da pesquisa, coleta de dados e análise dos dados; RO Martins participou da análise dos dados e da redação final do trabalho; ACL Dourado participou da análise dos dados e da redação final do trabalho; MTM Monteiro participou da coleta de dados e da análise dos dados; $\mathrm{D}$ Galato participou de todas as etapas da pesquisa como orientadora.

\section{Agradecimentos}

Agradecemos a Secretaria Municipal de Saúde de Tubarão e aos gestores das Unidades Básicas de Saúde pelo apoio na realização desta pesquisa. 


\section{Referências}

1. Farias AD, Cardoso MAA, Medeiros ACD, Belém LF, Simões MOS. Indicadores de prescrição médica nas unidades básicas de Saúde da Família no município de Campina Grande, PB. Rev Bras Epidemiol 2007; 10(2):149-156.

2. Silva T, Schenkel EP, Mengue SS. Nível de orientação a respeito de medicamentos prescritos a pacientes ambulatoriais de hospital universitário. Cad Saude Publica 2000; 16(2):449-455.

3. Organização Mundial da Saúde (OMS). Guia para a boa prescrição médica. Porto Alegre: Artmed; 1998.

4. World Health Organization (WHO). Adherence to long-term therapies: Evidence for action. Switzerland: WHO; 2003.

5. World Health Organization (WHO). How to investigate drug use in health facilities: selected drug use indicators. Genebra: WHO; 1993.

6. Menezes APS, Domingues MR, Baisch ALM. Compreensão das prescrições pediátricas de antimicrobianos em Unidades de Saúde em um município do sul do Brasil. Rev Bras Epidemiol 2009, 12(3):478-489.

7. Girotto E, Silva PV. A prescrição de medicamentos em um município do Norte do Paraná. Rev Bras Epidemiol 2006; 9(2):226-234.

8. Santos V, Nitrini SMOO. Indicadores do uso de medicamentos prescritos e de assistência ao paciente de serviços de saúde. Rev Saude Publica 2004; 38(6):819-826.

9. Silvério MS, Leite ICG. Qualidade das prescrições em município de Minas Gerais: uma abordagem farmacoepidemiológica. Rev Ass Med Bras 2010; 56(6):675-680.

10. Fröhlich SE, Mengue SS. Os indicadores de qualidade da prescrição de medicamentos da Organização Mundial da Saúde ainda são válidos? Cien Saude Colet 2011; 16(4):2289-2296.

11. Instituto Brasileiro de Geografia e Estatística (IBGE). Estimativas populacionais. [página na Internet] 2008. [acessado 2008 ago 12]. Disponível em: http://www. ibge.gov.br/cidade sat/topwindow.htm?1

12. Pan American Health Organization (PAHO). Rapid Pharmaceutical Management Assessment: an indicator based approach. Washington: PAHO; 1995.

13. Associação Nacional de Empresas e Pesquisas. Critério de Classificação Econômica Brasil. 2008 [acessado 2008 jul 10]. Disponível em: http://www.viver bem.fmb.unesp.br/docs/classificacaobrasil.pdf.

14. World Health Organization. Using indicators to measure country pharmaceutical situations: Fact book on WHO level I and II monitoring indicators. 2006 [cited 2012 Jul 28]. Available in: http://www.who.int/ medicines/publications/WHOTCM2006.2A.pdf.

15. Organización Mundial de la Salud (OMS). Promoción del uso racional de medicamentos: componentes centrales. Perspectivas políticas sobre medicamentos de la OMS sobre medicamentos. Ginebra: OMS; 2002.
16. Kawano DF, Pereira LRL, Ueta JM, Freitas O. Acidentes com medicamentos: como minimizá-los? Rev Bras Cienc Farm 2006; 42(4):487-495.

17. Oliveira CAP, Marin MJS, Marchioli M, Pizoletto BHM, Santos RV. Caracterização dos medicamentos prescritos aos idosos na Estratégia Saúde da Família. Cad Saude Publica 2009; 25(5):1007-1016.

18. Acurcio FA, Silva AL, Ribeiro AQ, Rocha NP, Silveira MR, Klein CR, Rozenfeld S. Complexidade do regime terapêutico prescrito para idosos. Rev Assoc Med Bras 2009; 55(4):468-474.

19. Brasil. Ministerio da Saúde (MS). Agência Nacional de Vigilância Sanitária. Decreto $n^{\circ} 3181$. Regulamenta a lei $n^{\circ} 9.797 / 1999$ que dispõe sobre a vigilância sanitária, estabelece o medicamento genérico, dispõe sobre a utilização de nomes genéricos em produtos farmacêuticos e dá outras providências. Diário Oficial da União 1999; 24 set.

20. Barie PS. Multidrug-resistant organisms and antibiotic management. Surg Clin North Am 2012; 92(2): 345-391.

21. Silva RM, Traebert J, Galato D. Kleibsiela penumoniae carbabenemase (KPC)-producing Kleibsiela pneumoniae: a review of epidemiological and clinical aspects. Expert Opin Biol Ther 2012; 12(6):663-671.

22. Fröhlich SE, Dal Pizzol TS, Mengue SS. Instrumento para avaliação do nível de conhecimento da prescrição na atenção primária. Rev Saude Publica 2010; 44(6):1046-1054.

23. Galato D, Silva ES, Tiburcio LS. Estudo de utilização de medicamentos em idosos residentes em uma cidade do sul de Santa Catarina (Brasil): um olhar sobre a polimedicação. Cien Saude Colet 2010; 15(6):2899-2905.

24. Follador W. Farmacoeconomia: promovendo o uso racional de medicamentos com qualidade de vida. In: Zubioli A, organizador. A farmácia clínica na farmácia comunitária. Brasília: Ethosfarma; 2001. p. 135-136.

25. Marin N, Luiza VL, Osório-de-castro CGS, Machado-dos-Santos S. Assistência farmacêutica para gerentes municipais. Rio de Janeiro: Organização Pan-Americana de Saúde (OPAS); 2003.

Artigo apresentado em 23/07/2012

Aprovado em 30/08/2012

Versão final apresentada em 03/09/2012 
